\title{
APRENDER A APRENDER: PROPOSTAS DE INTERVENÇÃO EM ESTRATÉGIAS DE APRENDIZAGEM ${ }^{1}$
}

\section{Evely Boruchovitch}

\section{RESUMO}

É inegável a escassez de propostas de intervenções e de elaboração de materiais instrucionais nacionais, relativos às estratégias de aprendizagem, em todos os segmentos da escolarização formal. O objetivo deste artigo é descrever e analisar alguns estudos nacionais, realizados e em andamento, tendo como referencial teórico a Psicologia Cognitiva baseada na Teoria do Processamento da Informação. Espera-se contribuir para discussão e produção de conhecimentos nessa área.

\section{PALAVRAS-CHAVE}

Estratégias de aprendizagem; Sessões de intervenção; Metacognição; Aprendizagem auto-regulada

\section{LEARNING TO LEARN: INTERVENTION PROPOSALS IN LEARNING STRATEGIES}

\begin{abstract}
The lack of national intervention proposals and instructional materials regarding learning strategies for school years is acknowledged by research. In line with that, the purpose of this paper is to describe and analyze carried out and ongoing national investigations adopting the Cognitive Psychology based on the Information Processing as a theoretical framework. There is a hope to contribute to the discussion and knowledge construction in this area.
\end{abstract}

\section{KEYWORDS}

Learning strategies; Intervention sessions; Metacognition; Self-regulated learning

\footnotetext{
${ }^{1}$ A autora agradece o apoio financeiro do CNPq e da Capes.
} 


\section{ARTIGO \\ Área Temática: Psicologia Educacional}

\section{INTRODUÇÃO}

A concepção de que a educação formal deve ser orientada para a promoção do estudante auto-regulado e responsável pela sua própria aprendizagem tem sido defendida, recentemente, por diversas abordagens teóricas (WOOLFOLK, 2000). A Psicologia Cognitiva baseada na Teoria do Processamento da Informação tem concentrado seus esforços em esclarecer como os seres humanos adquirem, armazenam e utilizam as informações. Adeptos desta visão destacam a importância de se transcender o ensino dos fatos e de se assegurar a apropriação, por parte dos estudantes, dos processos psicológicos pelos quais se aprendem os fatos. Acreditam no ensino de estratégias de aprendizagem como um caminho frutífero em direção à auto-regulação da aprendizagem (BORUCHOVITCH, 1993;1999; GRAHAM; HARRIS; MASON, 2005; POZO, 1996; PRESSLEY; LEVIN, 1983).

As estratégias de aprendizagem são seqüências de procedimentos empregadas para apoiar as três etapas fundamentais do processamento da informação: sua aquisição, seu armazenamento e sua utilização (NISBETT; SCHUCKSMITH; DANSEREAU, 1987 apud POZO, 1996). Podem também ser consideradas como qualquer procedimento adotado para a realização de uma determinada tarefa escolar (DA SILVA; DE SÁ, 1997). Estudiosos do tema as dividem e as classificam de várias maneiras, sendo mais comum a distinção entre as estratégias cognitivas e metacognitivas (DEMBO, 1994; GARNER; ALEXANDER, 1989). Dembo (1994) clarifica que as estratégias cognitivas operam diretamente sobre o material a ser aprendido, auxiliando o estudante a melhor processar a informação. Já as estratégias metacognitivas, segundo este autor, são procedimentos que o indivíduo emprega para planejar, monitorar e regular o seu próprio pensamento e ação. Uma análise mais detalhada do conceito de estratégias de aprendizagem, em nosso meio, pode ser encontrado em Boruchovitch (1999), Boruchovitch e Santos (2006) e Pozo (1996).

Que a intervenção em estratégias de aprendizagem melhora de forma expressiva o desempenho escolar dos alunos, em todos os segmentos da escolarização formal, tem sido um fato incontestável, evidenciado por um conjunto de pesquisas realizadas no estrangeiro, nas últimas décadas. Em linhas gerais, tem-se constatado que intervenções desta natureza, não só têm sido úteis para reduzir deficiências no processamento da informação em diversas áreas do saber, como matemática, leitura e escrita, mas também têm contribuído para a regulação dos aspectos afetivo-motivacionais relacionados com a aprendizagem dos estudantes, representando um meio de promover o aumento da capacidade do aluno de 
auto-regular a própria aprendizagem (GRAHAM; SCHWARTZ, 1993; GRAHAM; HARRIS; MASON, 2005; MACARTHUR; HATTIE; BIGGS; PURDUE, 1996; 1994; PRESSLEY; LEVIN, 1983; SOUVIGNIER; MOKHLESGERAMI, 2006).

\section{ALGUMAS CONSIDERAÇÕES SOBRE INTERVENÇÕES EM ESTRATÉGIAS DE APRENDIZAGEM}

As intervenções em Estratégias de Aprendizagem podem ser de vários tipos: cognitivas, metacognitivas, afetivas e mistas. As intervenções do tipo cognitivo são voltadas para o trabalho com uma ou mais estratégias de aprendizagem específicas (sublinhar, anotar). As do tipo metacognitivo são orientadas para apoiar os processos executivos de controle, como o planejamento, o monitoramento e a regulação dos processos cognitivos e do comportamento, já que o aumento do conhecimento metacognitivo vem sendo pensado como uma forma de se desenvolver o controle executivo. As intervenções do tipo afetivo destinam-se a controlar, modificar e eliminar estados internos do estudante, que possam ser incompatíveis com o bom processamento da informação. Em geral, predomina, nas investigações mais recentes, a intervenção do tipo mista, na qual atividades voltadas para o progresso cognitivo, o desenvolvimento metacognitivo, a promoção e a manutenção de um estado interno satisfatório para a aprendizagem são utilizadas de forma combinada. Segundo Hattie, Biggs e Purdue (1996), as intervenções de delineamentos mistos são as que mais se associam a resultados positivos na aprendizagem, nos diversos segmentos da escolarização.

Pressley et al. (1995) definiram nove pontos que devem ser levados em conta, ao se realizar uma intervenção em estratégias de aprendizagem, que serão brevemente descritos a seguir: ensinar somente uma estratégia de aprendizagem de cada vez, incluindo informações metacognitivas sobre o porquê, o onde e o quando aplicá-la; fornecer explicações pormenorizadas de cada nova estratégia, modelando-a; repetir a modelagem e a explicação, eliminando possíveis dúvidas; criar contextos diversificados, nos quais se possam praticar as estratégias ensinadas; incentivar o monitoramento da compreensão do estudante acerca de como está fazendo e o que está pensando, quando está usando as estratégias; prover oportunidades que favoreçam a capacidade de o estudante generalizar o uso das estratégias aprendidas para outras situações, nas quais elas sejam adequadas; aumentar a motivação dos estudantes e promover o processamento profundo e reflexivo da informação. Finalmente, Pressley et al. (1995) também destacam a importância de se aproveitarem todos os momentos para a realização de um trabalho orientado para a 
modificação de estados afetivo-motivacionais que possam ser prejudiciais à aprendizagem de qualidade. Almeida (2002) acrescenta que a intervenção em estratégias de aprendizagem deve ampliar o conhecimento dos alunos sobre elas, de modo a assegurar a flexibilidade no seu uso, bem como contribuir para aumentar o autoconhecimento e a autoregulação dos estudantes. Para maiores detalhes a respeito destes pontos, ver também Boruchovitch,Costa e Neves (2005).

\section{ALGUMAS PESQUISAS NACIONAIS RELATIVAS À INTERVENÇÃO EM ESTRATÉGIAS DE APRENDIZAGEM}

Como descrito em Boruchovitch (2006a), em nível nacional existem poucos estudos do tipo quase-experimental ou exploratórios, que visam verificar o efeito de intervenções em estratégias de aprendizagem no desempenho escolar de estudantes, tendo como referencial teórico a Psicologia Cognitiva baseada na Teoria do Processamento da Informação (COSTA, 2005; JALLES, 1997; MOLINA, 1983; 1984).

Na investigação de Jalles (1997), alunos da pré-escola, que receberam sessões de intervenção em estratégias de aprendizagem para a realização de uma tarefa que envolvia raciocínio espacial, apresentaram um percentual de erros significativamente menor do que o grupo de controle.

Pesquisas voltadas para a intervenção em habilidades de estudos específicas, como a leitura, realizadas com alunos da quinta à oitava série do ensino fundamental, atestaram uma melhora considerável no desempenho do aluno como decorrência da intervenção (MOLINA, 1983; 1984). Nesta mesma linha, Lopes (1997) realizou um estudo orientado para o desenvolvimento de estratégias metacognitivas, associadas à leitura de alunos de quinta série do ensino fundamental, que apresentavam problemas na leitura. Consistente com os estudos de Molina (1983; 1984), os resultados de Lopes (1997) apontaram para a melhoria da capacidade da leitura dos estudantes como decorrência da intervenção realizada.

No ensino médio, Carneiro e Aquino (1999) testaram a eficácia de um programa de promoção cognitiva com o objetivo de favorecer o rendimento escolar de alunos de uma escola agrotécnica, localizada no sul do Brasil. O “programa de promoção cognitiva” de Almeida e Morais (1989), desenvolvido em Portugal, foi utilizado nesta pesquisa. Os participantes (125) eram da primeira série do segundo grau, de ambos os sexos e com idades entre 13 e 20 anos. Os dados demonstraram que o programa foi eficiente, 
contribuindo para o desenvolvimento cognitivo e para a melhoria do rendimento acadêmico dos participantes.

Especificamente, em relação aos alunos dos cursos superiores, as pesquisas nacionais realizadas, relativas à intervenção em estratégias de aprendizagem, referem-se ao uso da metacognição em atividades de leitura em sala de aula e à intervenção em estratégias de estudo e de leitura (RODRIGUES, 1999; SAMPAIO; SANTOS, 2004; SANTOS, 1997; SILVA; SANTOS, 2004). Em linhas gerais, estes estudos atestam uma melhora expressiva no desempenho dos alunos que fizeram parte da intervenção.

Diferentemente dos estudos anteriormente mencionados, Darsie (1996) utilizou diários para registro da reflexão sobre a aprendizagem, como um exercício metacognitivo, para se avaliar a aprendizagem de alunos de um curso de formação de professores. Esta pesquisa concluiu pela necessidade de se converter a avaliação num instrumento de aprendizagem que possibilite a construção do conhecimento, mediante o exercício da metacognição, da auto-reflexão e da tomada de consciência da própria aprendizagem.

A seguir, será realizado um breve relato das propostas de intervenção em estratégias de aprendizagem, delineadas e desenvolvidas no Grupo de Pesquisa e Estudos em Psicopedagogia (GEPESP), do programa de Pós-Graduação da Faculdade de Educação da Universidade Estadual de Campinas.

\section{INTERVENÇÃO EM ESTRATÉGIAS DE APRENDIZAGEM: UMA BREVE DESCRIÇÃO DO TRABALHO REALIZADO NO GRUPO DE PESQUISAS E ESTUDOS EM PSICOPEDAGOGIA-GEPESP-UNICAMP}

Embora o trabalho com intervenção em estratégias de aprendizagem no GEPESP ainda se encontre na sua infância, pode-se constatar que alguns passos iniciais foram dados. Até o presente momento, três intervenções foram delineadas no GEPESP: uma voltada para a melhoria da escrita de alunos do ensino fundamental (COSTA, 2005; COSTA; BORUCHOVITCH, 2007a, 2007b), uma orientada para o desenvolvimento de estratégias auto-reguladas de leitura, também para alunos do ensino fundamental (GOMES; BORUCHOVITCH, 2003; GOMES; BORUCHOVITCH, 2007) e uma para melhorar as estratégias de aprendizagem de alunos de Curso de Pedagogia (NEVES; BORUCHOVITCH, 2004a, 2004b).

Costa (2005) conduziu uma intervenção em estratégias de aprendizagem em escrita, visando proporcionar uma qualidade maior na produção de textos narrativos de alunos da $6^{\mathrm{a}}$ série de uma escola pública de Catalão, Goiás. A intervenção consistiu de sete sessões 
de duração de 50 minutos cada, planejadas por Costa e Boruchovitch em 2002 (ver COSTA, 2005) tendo como base o modelo de auto-regulação proposto por Graham, Harris e MacArthur (1993). Foram trabalhadas estratégias específicas de produção de textos, como: pensar, planejar, escrever, escrever mais, revisar, reescrever, entre outras, bem como crenças dos alunos a respeito da inteligência, suas orientações motivacionais e metas, atribuições de causalidade e aspectos ligados à auto-instrução. Fizeram parte da amostra 35 alunos de ambos os sexos. Resultados revelaram que a intervenção foi muito útil para melhorar a produção de textos dos participantes (COSTA, 2005; COSTA; BORUCHOVITCH, 2007a, 2007b).

Gomes e Boruchovitch (2003) delinearam uma intervenção voltada para melhorar a compreensão da leitura de alunos da $4^{\mathrm{a}}$ série de uma turma do ensino fundamental de uma escola municipal de Jundiaí. A intervenção em estratégias auto-reguladas de leitura foi composta de sete sessões, de aproximadamente uma hora e meia de duração, com a freqüência de dois encontros semanais, durante um período de dois meses. As sessões foram organizadas de modo a promover a auto-regulação e a metacompreensão dos alunos em leitura de textos escritos. Incluíram o trabalho com estratégias de aprendizagem específicas para a melhoria da compreensão da leitura, nos três momentos (pré-leitura, durante a leitura e após a leitura), bem como atividades relativas a gerenciamento de tempo, à auto-avaliação quanto ao uso de estratégias de aprendizagem e a aspectos motivacionais e afetivos (PRESSLEY et al, 1995). A análise de seus efeitos encontra-se em andamento (GOMES; BORUCHOVITCH, 2007).

Numa tentativa de fortalecer a capacidade de processamento da informação do estudante de Curso de Pedagogia e lhe proporcionar possíveis conhecimentos para uma atuação profissional estratégica, Neves e Boruchovitch (2004 a) elaboraram uma intervenção, em caráter exploratório, cujo conteúdo foi dividido em três módulos, com duração de seis semanas, da seguinte forma: Módulo 1 - Aspectos motivacionais e afetivos na aprendizagem, Módulo 2 - O Processamento de Informação e as Estratégias de Aprendizagem e Módulo 3 - Estratégias de Aprendizagem: o sublinhar. Estes módulos instrucionais foram delineados, levando em conta a literatura da área (DARSIE, 1996; DEMBO, 2001; HATTIE; BIGGS; PURDUE, 1996). Toda a intervenção foi planejada para ser transmitida por meio de slides (arquivos ppt e html) e pela troca de informações entre os alunos e o professor, mediante os recursos do ambiente TelEduc. 
Vale ressaltar que as três propostas anteriormente relatadas apresentam alguns aspectos em comum. Todas foram inicialmente pré-testadas por estudos-piloto (em amostras que muito se assemelharam aos participantes) e refinadas, sempre que necessário. Acresce-se que o trabalho focado nos conteúdos relativos às estratégias de aprendizagem específicas foi conduzido numa dupla vertente, isto é, não só teórica, mas também vivencial, metacognitiva e auto-reflexiva. Existiu ainda nestas propostas uma preocupação em se atuar nos componentes afetivos e motivacionais que se associam ao emprego efetivo de estratégias de aprendizagem; preocupação que, segundo a literatura da área, muito contribui para melhorar a qualidade e potencializar os efeitos de uma intervenção (DARSIE, 1996; DEMBO, 2001; HATTIE; BIGGS; PURDUE, 1996; PRESSLEY et al., 1995). Todas as três propostas envolveram a utilização de alguns instrumentos e materiais que foram desenvolvidos e ou adaptados no GEPESP, à luz da Psicologia Cognitiva baseada na Teoria do Processamento da Informação e das Teorias Sociocognitivas da Motivação para esta, dentre outras finalidades (BORUCHOVITCH, 2006b).

\section{CONSIDERAÇÕES FINAIS}

Entre as principais contribuições da Psicologia Cognitiva baseada na Teoria do Processamento da Informação para a educação, podem-se destacar a convicção de que todo aluno pode ser bem-sucedido e auto-regulado (ZIMMERMAN; BONNER e KOVACH, 1996), a consciência de que "ensinar a aprender" e "aprender a aprender" são projetos pedagógicos difíceis, porém passíveis de serem alcançados (PRESSLEY; BORKOWSKI; SCHNEIDER, 1989), e a crença nas estratégias de aprendizagem como variável-chave para o fortalecimento da capacidade de aprender e de elevar a qualidade do desempenho escolar de alunos (HATTIE; BIGGS; PURDUE, 1996).

Alunos de baixo rendimento escolar podem beneficiar-se muito de intervenções em estratégias de aprendizagem (BORUCHOVITCH, 1993). Podem aprender a ampliar anotações de aulas, sublinhar partes relevantes de um texto, monitorar a compreensão na hora da leitura, usar técnicas de memorização, fazer resumos, planejar, controlar cognições negativas e estados afetivo-motivacionais disfuncionais, entre outras estratégias. Resultados de pesquisas, quer em nível internacional, quer em nível nacional, evidenciam que a intervenção em estratégias de aprendizagem tem sido frutífera, em linhas gerais, gerando avanços no uso das estratégias envolvidas, bem como melhorando o rendimento escolar e os aspectos afetivo-motivacionais dos alunos (COSTA, 2005; HATTIE; BIGGS; 
PURDIER, 1996; JALLES, 1997; LOPES, 1997; RODRIGUES, 1999; SANTOS, 1997; SAMPAIO; SANTOS, 2004; SILVA; SANTOS, 2004).

Sem dúvida, os resultados das investigações em intervenção em estratégias de aprendizagem são animadores, indicando o seu caráter promissor, já que, como mencionado em Boruchovitch, Costa e Neves (2005), evidências, em nível nacional, sugerem que mesmo os estudantes universitários apresentam um repertório de estratégias de aprendizagem deficitárias e dificuldades de processamento adequado da informação, em situações de estudo e aprendizagem, por diversas razões.

Se, por um lado, sabemos que vivemos um cenário educacional no qual intervir em estratégias de aprendizagem se encontra ainda incipiente, mas parece poder muito contribuir para atenuar os problemas existentes, por outro lado, precisamos transcender abordagens remediativas e adotarmos um enfoque preventivo no qual o aprender a aprender seja possível desde o início da escolarização formal (BORUCHOVITCH, 2006a).

Que o conhecimento construído a partir das intervenções realizadas, em andamento e ainda por ocorrer, possa ser utilmente convertido em iniciativas direcionadas para o desenvolvimento de propostas curriculares mais preventivas, no que concerne à promoção da capacidade do aluno para aprender a aprender, tornar-se um estudante bem sucedido e responsável pela sua própria aprendizagem.

\section{REFERÊNCIAS}

ALMEIDA, L. S. Facilitar a aprendizagem: ajudar os alunos a aprender e a pensar. Psicologia Escolar e Educacional. Campinas, v. 6, n. 2, p. 155-165, 2002.

ALMEIDA, L.; MORAIS, M. F. Programa "promoção cognitiva". Revista Portuguesa de Educação, Braga, v. 2, n 1, p. 25-32, 1989.

BORUCHOVITCH, E. A psicologia cognitiva e a metacognição: novas perspectivas para o fracasso escolar brasileiro. Tecnologia Educacional, Rio de Janeiro, v. 22, n.110-111, p. 22-28, 1993.

As estratégias de aprendizagem e o desempenho escolar de crianças brasileiras: considerações para a prática educacional. Psicologia: Reflexão e Crítica, Porto Alegre, v.12, n. 2, p. 361-376, 1999.

Estratégias de aprendizagem: uma análise à luz das variáveis demográficas e motivacionais. 2006a. Tese (Livre Docência) - Faculdade de Educação, Universidade Estadual de Campinas, Campinas, 2006a. 
Avaliação psicoeducacional: desenvolvimento de instrumentos à luz da psicologia cognitiva baseada na Teoria do Processamento da Informação. Avaliação Psicológica, São Paulo, v. 5, n. 2, p. 145-152, 2006 b.

A metacognição e a construção do conhecimento: sua importância na formação de professores. In: CONGRESSO NACIONAL DE PSICOLOGIA ESCOLAR E EDUCACIONAL, 8; SIMPÓSIO CRIATIVIDADE, METACOGNIÇÃO E APRENDIZAGEM À DISTÂNCIA NA GESTÃO DO CONHECIMENTO. 2007, São João Del Rei [Anais...] João Del Rei, 2007. CD-ROM, ISSN 1981-2566.

BORUCHOVITCH, E; COSTA, E. R.; NEVES, E. R. C. Estratégias de aprendizagem: contribuições para a formação de professores nos cursos superiores. In: JOLY, M.C.; SANTOS, A. A. A.; SISTO, F. F (Org.). Questões do cotidiano universitário. São Paulo: Casa do Psicólogo, 2005. p. 239-260.

BORUCHOVITCH, E.; SANTOS, A. A. A. Estratégias de aprendizagem: conceituação e avaliação. In: NORONHA, A. P. P.; SISTO, F. F. (Org.). Facetas do fazer em avaliação psicológica. São Paulo: Vetor, 2006. p. 107-124.

CARNEIRO, E. G.; AQUINO, S. (Org.). Uma experiência brasileira de promoção cognitiva. In: da REUNIÃO ANUAL DE PSICOLOGIA - SBP, 24, Campinas, 1999. [Anais...] Campinas p. 47.

COSTA, E. R. A intervenção por meio da instrução em estratégias de aprendizagem: contribuições para a produção de textos. 2005. Tese (Doutorado em Educação) Faculdade de Educação, Universidade Estadual de Campinas, Campinas, 2005.

COSTA, E. R.; BORUCHOVITCH, E. Intervenção em estratégias de aprendizagem em sala de aula: avaliando os elementos básicos de uma narrativa. In: CONGRESSO NACIONAL DE PSICOLOGIA ESCOLAR E EDUCACIONAL, 8, São João Del Rei [Anais...] São João Del Rei, 2007a. CD-ROM. ISSN 1981-2566.

COSTA, E. R.; BORUCHOVITCH, E. A produção de textos narrativos e o ensino de estratégias de aprendizagem. In: CONGRESSO NACIONAL DE PSICOLOGIA ESCOLAR E EDUCACIONAL, 8, São João Del Rei [Anais...] São João Del Rei, 2007b. CD-ROM. ISSN 1981-2566.

DA SILVA A. L.; DE SÁ, L. Saber estudar e estudar para saber. Porto, Portugal: Porto Ed., 1997. Coleção Ciências da Educação.

DARSIE, M. M. P. Avaliação e aprendizagem. Cadernos de Pesquisa, São Paulo, v. 99, n. 11, p. 47-59, 1996.

DEMBO, M. H. Applying educational psychology. 5. ed. New York: Longman Publishing Group. 1994. 
DEMBO, M. H. Learning to teach is not enough: future teachers also need to learn to learn. Teacher Education Quaterly, California, v. 28, n. 4, p. 23-35, 2001.

GARNER, R.; ALEXANDER, P. A. Metacognition: Answered and unanswered questions. Educational Psychologist, New Jersey, v. 24, n. 2, p. 143-158, 1989.

GOMES, M. A. M.; BORUCHOVITCH, E. Procedimentos de Intervenção em Estratégias de Aprendizagem para a Compreensão Auto-Regulada em Leitura. Programa de Pós-Graduação em Educação, Faculdade de Educação, Universidade Estadual de Campinas, 2003. (Trabalho não publicado).

GOMES, M. A. M.; BORUCHOVITCH, E. Ensino de estratégias de aprendizagem e o desenvolvimento da compreensão leitora. In: CONGRESSO NACIONAL DE PSICOLOGIA ESCOLAR E EDUCACIONAL, 8, São João Del Rei [Anais...] São João Del Rei, 2007b. CD-ROM. ISSN 1981-2566.

GRAHAM, S.; HARRIS, K.R.; MASON, L. Improving the writing performance, knowledge, and self-efficacy of struggling young writers: the effects of self-regulated strategy development. Contemporary Educational Psychology, Maryland, v. 30, p. 207241, 2005.

HATTIE, J.; BIGGS, J.; PURDIER, N. Effects of learning skills interventions on students: a meta analysis._Review of Educational Research, Washington, v. 66, n. 2, p. 99-136, 1996.

JALLES, C. M. C. R. O efeito de instruções sobre estratégias metacognitivas de crianças pré-escolares em solução de problema geométrico. 1997. Dissertação (Mestrado em Educação) - Faculdade de Educação, Universidade Estadual de Campinas, Campinas, 1997.

LOPES, M. C. C. O uso de estratégias cognitivas e metacognitivas no ensino/ aprendizagem da leitura no $1^{\circ}$ Grau: uma proposta de intervenção. 1997. Dissertação (Mestrado em Lingüística Aplicada) - Instituto de Estudos da Linguagem, Universidade Estadual de Campinas, Campinas, 1997.

MACARTHUR; GRAHAM, S. ; SCHWARTZ, S. Integrating strategy instruction and word processing into a process approach to writing instruction. School Psychology Review, Bethesda, v. 23, n. 4, 1993.

MOLINA, O. Desenvolvimento de habilidades de estudo: uma estratégia ao alcance do professor. Educação e Seleção, Rio de Janeiro, v. 8, p. 45-53, 1983.

Diferenças no desempenho em leitura com resultado de treinamento em habilidades de estudo. Educação e Seleção, Rio de Janeiro, v. 10, p. 35-42, 1984.

NEVES, E. R. C.; BORUCHOVITCH, E. Módulos instrucionais em estratégias de aprendizagem e aspectos afetivo-motivacionais para intervenção com alunos universitários por meio da Educação a Distância -. Programa de Pós-Graduação em 
Educação, Faculdade de Educação, Universidade Estadual de Campinas, 2004a. (Trabalho não publicado).

NEVES, E. R. C.; BORUCHOVITCH, E. Estratégias de aprendizagem para alunos universitários por meio da Educação à Distância. Caderno de Resumos da Divulgação de Ações do Ministério de Educação sobre Educação a Distância. Agência de Inovação da UNICAMP-INOVA, Campinas, p. 3, 2004b.

NISBET, J.; SCHUCKSMITH, J. Estratégias de Aprendizaje. Madrid: Santillana, 1986.

POZO, J. I. Estratégias de Aprendizagem. In: COLL, C.; PALÁCIOS, J.; MARCHESI, A. (Ed.). Desenvolvimento psicológico e educação: psicologia da educação. Porto Alegre: Artes Médicas, 1996. p. 176-197.

PRESSLEY, M.; LEVIN, J. R. Cognitive strategy research: psycological foundations. New York: Springer-Verlag, 1983.

PRESSLEY, M.; BORKOWSKI, J. G.; SCHNEIDER, W. Good information processing: what it is and how education can promote it. Journal of Educational Research, Washington, v. 13, n. 8, p. 857-867, 1989.

PRESSLEY, M. et al. Cognitive strategy instruction that really improves children's academic performance. 2 ed. Cambridge, Massachuttes: Brookline Books, 1995.

RODRIGUES, L. A. D. Estratégias de aprendizagem na aula de língua estrangeira: a busca da sintonia entre o ensinar e o aprender. Dissertação (Mestrado em Educação) Faculdade de Educação, Universidade Estadual de São Paulo, São Paulo, 1999.

SAMPAIO, I. S.; SANTOS, A. A. A. Leitura e redação entre universitários: avaliação de um programa de intervenção. Psicologia em Estudo, Maringá, v. 7, n. 1, p. 1-18, 2002.

SANTOS, A. A. A. Psicopedagogia no $3^{\circ}$ grau: avaliação de um programa de remediação em leitura e estudo. Proposições, Campinas, v. 8, n. 1, p. 27-37, 1997.

SILVA, M. J. M.; SANTOS, A. A. A. A avaliação da compreensão em leitura e o desempenho acadêmico de universitários. Psicologia em Estudo, Maringá, v. 9, n. 3, p. 121, 2004.

SOUVIGNIER, E.; MOKLESGERAMI, J. Using self-regulation as a framework for implementing strategy instruction. Learning and Instruction, Grécia, n. 16, p. 57-71, 2006.

WOOLFOLK, A. Psicologia da educação. Porto Alegre: Artes Médicas, 2000.

ZIMMERMAN, B. J.; BONNER, S.; KOVACH, R. Developing self-regulated learners: Beyond achievement to self-efficacy. Washington: American Psychology Association, 1996. 
Faculdade de Educação da Universidade Estadual de Campinas

(UNICAMP); Membro do Grupo de Estudos e Pesquisas em

Psicopedagogia (GEPESP) do Programa de Pós Graduação em

Educação da UNICAMP.

E-mail: evely@unicamp.br 